\title{
Multi-expert Seal Imprint Verification System for Bankcheck Processing
}

\author{
Katsuhiko Ueda and Ken'ichi Matsuo \\ Department of Information Engineering, Nara National College of Technology \\ Yamatokoriyama, Nara 639-1080, Japan \\ ueda@info.nara-k.ac.jp
}

\begin{abstract}
A difficult problem encountered in automatic seal imprint verification is that the system is required an extremely low error rate despite of the variety of seal imprint quality. To conquer this problem, we propose a multi-expert seal imprint verification system, which combines two different verification algorithms. The first verification algorithm is based on a method using local and global features of seal imprint. The second one uses a special correlation method based on a global approach. The two algorithms are combined by a voting strategy. Experimental results showed that the combination of the two algorithms improves significantly the verification performance both on "false-acceptance error rate" and "false-rejection error rate".
\end{abstract}

\section{Introduction}

Automatic bankcheck processing is an active topic in the field of document analysis and processing. Validity confirmation of bankchecks is one of the important problems in automatic bankcheck processing. In Japan and some oriental countries, seal imprints have been widely used for validating bankchecks.

Many researches on this problem have been made. However the design of seal imprint verification system for validating bankchecks still remains a difficult problem because of some specific characteristics: (1) forged seal imprints with very similar pattern to a registered one may appear in practical applications. (2) seal imprints may have a lot of variations, even if they are all produced from a single seal. As for (1), Ueda [1] proposed a verification algorithm using local and global features of seal imprints (called "feature-based algorithm" in this paper). As for (2), Horiuchi[3] proposed an algorithm using a special correlation method (called "correlation-based algorithm" in this paper). However it is difficult to adapt to all of the above specific characteristics by only individual verification algorithms.

In this paper, we propose a new multi-expert seal imprint verification system, which combines two different verification algorithms mentioned above. Through the verification experiments, we will also present that the combination of the two verification algorithms allows a significant improvement in the verification performance.

D. Lopresti, J. Hu, and R. Kashi (Eds.): DAS 2002, LNCS 2423, pp. 62-65 2002.

(C) Springer-Verlag Berlin Heidelberg 2002 


\section{Verification Scheme}

The seal imprint verification system presented in this paper is shown in Fig.1. The seal imprint is first extracted from a bankcheck image by a color clustering technique 2]. Successively, the extracted seal imprint is positioned over the corresponding registered seal imprint $[1$. Each verification module verifies the seal imprint with the corresponding registered one. The system provides the response on the authenticity of bankcheck by combining the outputs of both verification modules.

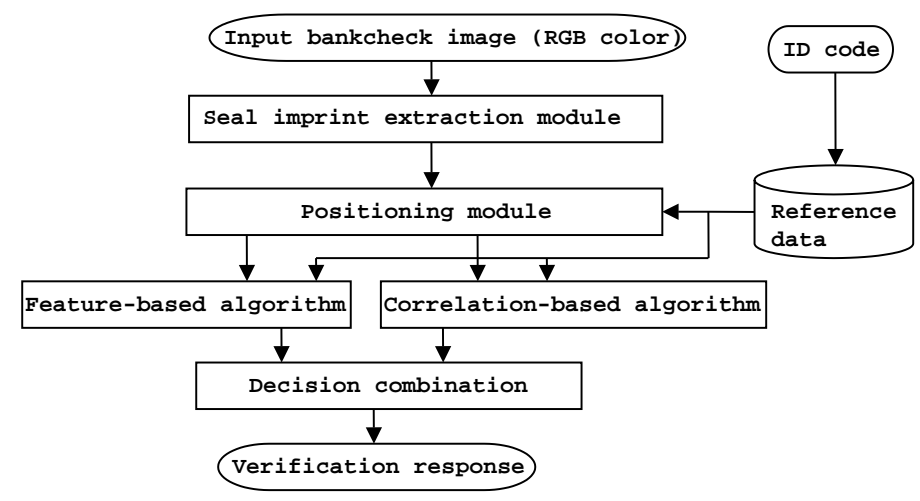

Fig. 1. Multi-expert seal imprint verification system.

\subsection{Feature-Based Algorithm[1, [2]}

This module verifies seal imprints using their local and global features. The dissimilarity measure for verifying seal imprints is computed by using the features. Let $\left\{D_{i}(i=1,2, \ldots, n)\right\}$ be the obtained dissimilarities between the registered seal imprint and its $n$ genuine samples for training. Moreover, let $D_{a v}$ and $\sigma_{I}$ be the mean value and the standard deviation of $\left\{D_{i}\right\}$ respectively. The dissimilarity limit (threshold) $D_{T}$ of the genuine seal imprints for each registered one is defined as the following equation.

$$
D_{T}=D_{a v}+a \sigma_{I}
$$

where " $a$ " is a certain constant value.

After the dissimilarity $D_{u}$ of an examined seal imprint is computed, two types of decision can be made as follows:

1. If $D_{u} \leq D_{T}$, the input seal imprint is classified as a genuine.

2. If $D_{u}>D_{T}$, the input seal imprint is classified as a forgery. 


\subsection{Correlation-Based Algorithm $[3$}

The dissimilarity measure between an examined and a registered seal imprints is defined as follows:

$$
D=1-\frac{\sum_{x, y} f(x, y) g(x, y)}{\sum_{x, y} f(x, y)}
$$

where, $f(x, y), g(x, y)$ denote an examined and a registered seal imprint image respectively. Both images are binary. Equation (2) means that this correlation algorithm evaluates only the excess of pixels of an examined seal imprint. The methods of a threshold definition and a decision are the same as those described in the above subsection.

\subsection{Decision Combination Module}

The problem of seal imprint verification has specific characteristics as described in Section 1. The systems in practical applications are required an extremely low error rate (especially "false acceptance error rate"), even if a considerable rate of rejection is acceptable. Combination methods that require training are not useful, because a large number of training samples are not available. Moreover each verification module gives a verification result in the form of a Boolean value ("genuine" or "forgery"). Therefore, in this work, a majority voting method has been adopted to combine the two verification algorithms. Specifically, the examined seal imprint is classified as "genuine", if the results of both verification algorithms are genuine. It is classified as "forgery", if the results of both verification algorithms are forgery. Otherwise it is rejected as "ambiguous".

\section{Experimental Results}

We used five genuine seals with various shapes and three forged seals for each genuine one. The example of seal imprints generated from each seal is shown in Fig.2. 100 imprints for each genuine seal and 50 imprints for each forged seal were stamped on real bankchecks. Therefore, 500 genuine seal imprints and 750 forged seal imprints were used for the performance evaluation of this system.

The performance of this system were measured by verification experiment. The verification performance of the combination system as well as the performance of individual algorithms is compiled in Table 1. In this Table, FAR and FRR mean "false-acceptance error rate" and "false-rejection error rate" respectively. Rejection rate means "ambiguous rate" caused by rejection due to discrepancy between two verification results from individual verification algorithms. This result shows the different behavior of two verification algorithms, i.e., the feature-based algorithm performs better for detecting forgeries, while the correlation-based algorithm performs better for accepting genuine seal imprints. The proposed combination system allows a significant improvement in the verification performance. In fact, this system was able to achieve $\mathrm{FAR}=0.4 \%$, $\mathrm{FRR}=1.2 \%$, with Rejection rate of $17.1 \%$. 


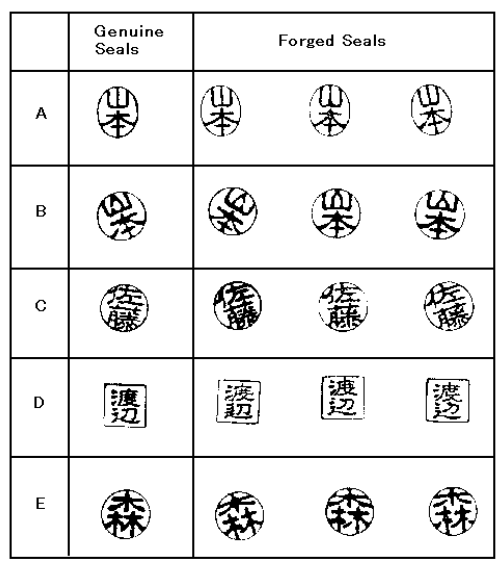

Fig. 2. Example of seals used in the experiments.

Table 1. Performance of the proposed system and individual verifiers.

\begin{tabular}{|c|c|c|c|}
\hline & $\begin{array}{c}\text { Feature-based } \\
\text { Algorithm }\end{array}$ & $\begin{array}{c}\text { Correlation-based } \\
\text { Algorithm }\end{array}$ & Proposed system \\
\hline FAR(\%) & 2.9 & 9.3 & $\mathbf{0 . 4}$ \\
\hline FRR(\%) & 11.4 & 5.6 & $\mathbf{1 . 2}$ \\
\hline Rejection Rate(\%) & - & - & $\mathbf{1 7 . 1}$ \\
\hline
\end{tabular}

\section{Conclusions}

In this paper, we proposed a multi-expert seal imprint verification system, which combines two different verification algorithms. The experimental results showed that the combination of the two different verification algorithms improves significantly the verification performance. This system might be feasible for practical applications.

Acknowledgement. This work was supported in part by Grant-in-Aid for Scientific Research under Grant No. 13680500 sponsored by Japan Society for the Promotion of Science.

\section{References}

1. K. Ueda, "Automatic seal imprint verification system with imprint quality assessment function", IEICE Trans. Inf. \& Syst., E77-D, 8, pp.885-894 (1994).

2. K. Ueda, T. Mutoh and K. Matsuo, "Automatic verification system for seal imprints on Japanese bankchecks", Proc. of ICPR'98, pp.629-632 (1998).

3. T. Horiuchi, "Automatic seal verification by Evaluating Positive Cost", Proc. of ICDAR'01 (2001). 